\title{
Concurrent germline BRCA1, BRCA2, and CHEK2 pathogenic variants in hereditary breast cancer: a case series
}

\author{
Jasmine Sukumar $^{1} \cdot$ Mahmoud Kassem $^{1} \cdot$ Doreen Agnese $^{3} \cdot$ Robert Pilarski $^{2} \cdot$ Bhuvaneswari Ramaswamy $^{1}$. \\ Kevin Sweet $^{2} \cdot$ Sagar Sardesai $^{1}$ (i)
}

Received: 30 November 2020 / Accepted: 5 January 2021 / Published online: 28 January 2021

(c) The Author(s) 2021

\begin{abstract}
Background Concurrent germline (g) pathogenic variants related to hereditary breast cancer represent a rare occurrence. While double heterozygosity in gBRCA1 and gBRCA2 has been reported in the past, herein we describe the first case of three known concurrent pathogenic variants identified in a family with a strong history of breast cancer.

Case presentation

The proband is a 55-year-old female diagnosed with synchronous bilateral breast cancers. She underwent a multi-gene panel testing indicating the presence of 3 concurrent heterozygous germline deleterious variants in BRCA1 (c.181T>G), BRCA2 (c.4398_4402delACATT), and CHEK2 (1100delC). The patient's two daughters (34 and 29 years-old) were found to be transheterozygous for inherited pathogenic variants in BRCA1 (c.181T>G) and CHEK2 (1100delC) genes.

Conclusion The cancer risk and phenotypic manifestations associated with transheterozygous or multiple concurrent deleterious germline variants in hereditary breast cancer requires further investigation. A personalized approach to counseling, screening, and risk reduction should be undertaken for these individuals.
\end{abstract}

Keywords BRCA1 $\cdot$ BRCA2 $\cdot$ CHEK2 $\cdot$ DNAI1 $\cdot$ Germline $\cdot$ Genetic $\cdot$ Variants $\cdot$ Breast cancer

\section{Introduction}

Germline (g) variants in breast cancer susceptibility genes are a significant risk factor for the development of breast cancer $[1,2]$ and as much as $10-15 \%$ of breast cancer cases are hereditary $[3,4]$. This includes both rare, high penetrance variants (e.g., $B R C A$ ) and rare, moderate risk variants (e.g., $C H E K 2$ ) [5, 6]. Clinical features associated with hereditary cancers such as early age of disease onset, family history of breast and ovarian cancer, bilateral breast cancer, male breast cancer, and Ashkenazi Jewish ancestry should prompt referral for genetic counseling $[2,7]$. Understanding

Sagar Sardesai

Sagar.Sardesai@osumc.edu

1 Division of Medical Oncology, The Ohio State University Wexner Medical Center, 1204A Lincoln Tower, 1800 Cannon Dr., Columbus, OH 43210, USA

2 Division of Human Genetics, The Ohio State University Wexner Medical Center, Columbus, OH, USA

3 Division of Surgical Oncology, The Ohio State University Wexner Medical Center, Columbus, OH, USA the precise risks and clinical implications associated with pathogenic variants is critical to establish best practices in screening and prevention and thereby improve outcomes for patients who are predisposed to hereditary breast cancer.

Of the known pathogenic variants related to hereditary breast cancer, those in the Breast Cancer Susceptibility Genes 1 and $2(B R C A 1 / 2)$ account for $50-60 \%$ of cases [1, 8]. Additionally, germline $B R C A 1$ pathogenic variants are more often associated with triple negative breast cancers with worse prognosis [9]. BRCA1/2 pathogenic variants account for approximately $1-2 \%$ of all breast cancer cases and the prevalence of $B R C A 1 / 2$ pathogenic variants in the general population is about 1 in 400 individuals [10]. However, the prevalence in the Ashekanzi Jewish population is higher at about 1 in 40 individuals, or 2 to $2.5 \%$ [11, 12]. BRCA1/2 genes code for tumor suppressor proteins involved in DNA double strand break repair; these proteins play a critical role in response to maintenance of genetic integrity. Aberration of BRCA proteins involved in the DNA repair pathway leads to homologous recombination deficiency and diminished response to DNA damage, thereby contributing to carcinogenesis [13]. BRCA1/2 pathogenic variants 
demonstrate high penetrance with approximately 50-90\% lifetime risk of breast cancer. In addition, $B R C A 1 / 2$ variants can increase the risk of second primary breast cancer $[2,14$, 15] and other solid tumors such as high-grade serous ovarian cancers, pancreatic adenocarcinoma, and melanomas [14].

Hereditary breast cancer is also caused by pathogenic variants in other well-reported susceptibility genes such as ATM, CDH1, CHEK2, PALB2, PTEN, TP53, and NF1. $C H E K 2$ (cell cycle checkpoint kinase 2) is a moderately penetrant breast cancer susceptibility gene which can increase the breast cancer risk by two to threefold [16-18]. The prevalence of CHEK2 pathogenic variants in the general population is approximately 1\% [5]. While deleterious CHEK2 mutations are reported at a higher frequency in individuals of Northern [19] and Eastern European descent [20], the exact prevalence in these populations compared with the general population is not clearly quantified. However, in one Polish study of 7494 breast cancer patients and 4346 controls, a CHEK2 pathogenic variant was detected in 3\% of women with breast cancer and $0.8 \%$ of women in the control group [20]. The CHEK2 gene codes for a serine/threonine protein kinase called Chk2 kinase which activates multiple proteins critical in maintenance of the cell cycle and DNA repair. This includes the tumor suppressor protein p53 which has an integral role in cell cycle control and apoptosis. Additionally, Chk2 kinase activates the BRCA1 protein, and this interaction is important in the normal function of cellular DNA damage response [21, 22]. Therefore, CHEK2 and BRCA are closely interrelated in the DNA break repair pathway and work together to maintain genomic integrity. The most common variant in the CHEK2 gene is the frameshift mutation 1100delC which codes for a truncated protein. This pathogenic variant is associated with a $28-37 \%$ lifetime risk of breast cancer, with the higher risk in those with strong family history $[2,20]$. CHEK2 pathogenic variants are also implicated in a 1.5-2 fold increase in colon cancer risk [23].

There is a paucity of data on the risk and clinical implications of an individual carrying multiple simultaneous pathogenic variants. The presence of co-existing $B R C A 1 / 2$ variants as well as concomitant $B R C A$ and $C H E K 2$ variants in an individual is exceedingly infrequent but has been described in the literature [24-26]. However, to the best of our knowledge, there are no prior reports of three simultaneous pathogenic germline variants contributing to hereditary breast cancer and we describe the first reported case.

\section{Proband and family case presentation}

The proband is a 55-year-old pre-menopausal Caucasian female with a past medical history of appropriately treated chronic hepatitis $\mathrm{C}$ infection, cirrhosis, coronary artery disease, and hyperthyroidism who was diagnosed with left breast cancer in August 2018. She underwent bilateral mastectomy and left axillary sentinel lymph node biopsy with bilateral reconstruction. Pathology revealed a $1.3 \mathrm{~cm}$, grade 3 invasive ductal carcinoma which was estrogen receptor negative $(0 \%)$, progesterone receptor weakly positive $(10 \%)$, and Her-2/neu negative (IHC Score 0). She was pathologically staged as $1 \mathrm{~A}$ (pT1cN0). An incidental right invasive ductal carcinoma was additionally found on review of the pathologic specimen, which was $0.3 \mathrm{~cm}$, grade 1 and strongly hormone receptor positive (estrogen receptor $100 \%$, progesterone receptor $100 \%$ ) and Her-2/neu negative (IHC score $1+$ ). Systemic adjuvant chemotherapy was recommended for the left breast cancer but the patient declined. She started adjuvant endocrine therapy with tamoxifen.

Notably, the proband endorsed a strong family history of breast cancer including a mother diagnosed at the age of 42 (bilateral, synchronous primaries), a sister at the age of 35, and a maternal grandmother at the age of 43 . Family history was also significant for melanoma in her brother. There was no family history of ovarian cancer. To her knowledge, none of her relatives had previously completed genetic testing. The patient was referred to a licensed genetic counselor for cancer genetic risk assessment and pre-test genetic counseling. Blood-based multi-gene germline testing was performed using an 11-gene panel that included ATM, BRCA1, BRCA2, CDH1, CHEK2, NBN, NF1, PALB2, PTEN, STK11, and TP53. The results indicated presence of three concurrent heterozygous germline pathogenic variants in BRCAI (c.181T>G), BRCA2 (c.4398_4402delACATT), and CHEK2 (1100delC). The proband received post-test genetic counseling and was advised on her cancer risk and risk reduction recommendations, as well as the potential inherited risk to her relatives. She elected to undergo laparoscopic bilateral salpingo-oophorectomy for ovarian cancer risk reduction, and pathology from this surgery was benign.

The patient's 34-year-old daughter, a pre-menopausal female with a past medical history significant for bipolar disorder, was referred for genetic counseling for her 50\% risk for each of the three separate pathogenic variants identified in her mother. There was no known family history of cancer in her father. She elected to pursue genetic testing for the 3 genes (BRCA1, BRCA2, CHEK2) which revealed a transheterozygous inheritance of pathogenic variants in the BRCA1 (c.181T $>G$ ) and CHEK2 (1100delC) genes, but did not report the $B R C A 2$ pathogenic variant identified in her mother. In October 2019, she had her first screening breast MRI and was found to have a suspicious mass in the right breast; subsequent biopsy revealed right invasive ductal carcinoma. She underwent a right radioactive seedlocalized lumpectomy and sentinel lymph node biopsy in December 2019 demonstrating two foci of grade 3 invasive ductal carcinoma $(0.7 \mathrm{~cm}$ and $0.25 \mathrm{~cm})$ with negative margins and negative sentinel lymph nodes, estrogen receptor 
low-positive (5\%), progesterone receptor negative $(0 \%)$, and Her-2/neu negative (IHC score 1+). She subsequently received adjuvant chemotherapy. However, she declined adjuvant endocrine therapy due to concern for possible side effects. Almost a year from diagnosis, she underwent a risk-reducing bilateral skin sparing mastectomy followed by reconstruction.

Based upon the described family history and genetic results, the proband's 29-year-old daughter underwent genetic testing. She had a different father than her 35-yearold half-sister, and to the proband's knowledge, there was no paternal family history of cancer. The results of genetic testing demonstrated the inheritance of pathogenic variants in BRCA1 (c.181T $>G)$ and CHEK2 (1100delC) genes. She has since established care in our institutional high-risk breast cancer program to undergo annual bilateral screening mammograms and bilateral breast MRIs. She declined chemoprophylaxis with endocrine therapy. A family pedigree is provided in Fig. 1.

\section{Discussion}

We describe the first reported case of three concurrent pathogenic germline variants in BRCA1, BRCA2, and CHEK2 associated with inherited breast cancer in an individual. Given the presence of 3 co-existing pathogenic variants in the proband, her family members were at a very high risk of carrying at least one moderate or high penetrance variant. Upon further genetic testing, we identified co-existing germline CHEK2 and BRCA pathogenic variants in this family cluster with breast cancer; this represents a rare occurrence with only few reported cases.

In review of the literature, including case reports [25-27] as well as public registries of human genetic variants [24, 28], simultaneous pathogenic variants in two different genes (referred to as double heterozygosity or transheterozygosity) related to hereditary breast cancer is an extremely uncommon occurrence. For example, there have been less than 100 reported cases of co-existing $B R C A 1 / 2$ variants; this is

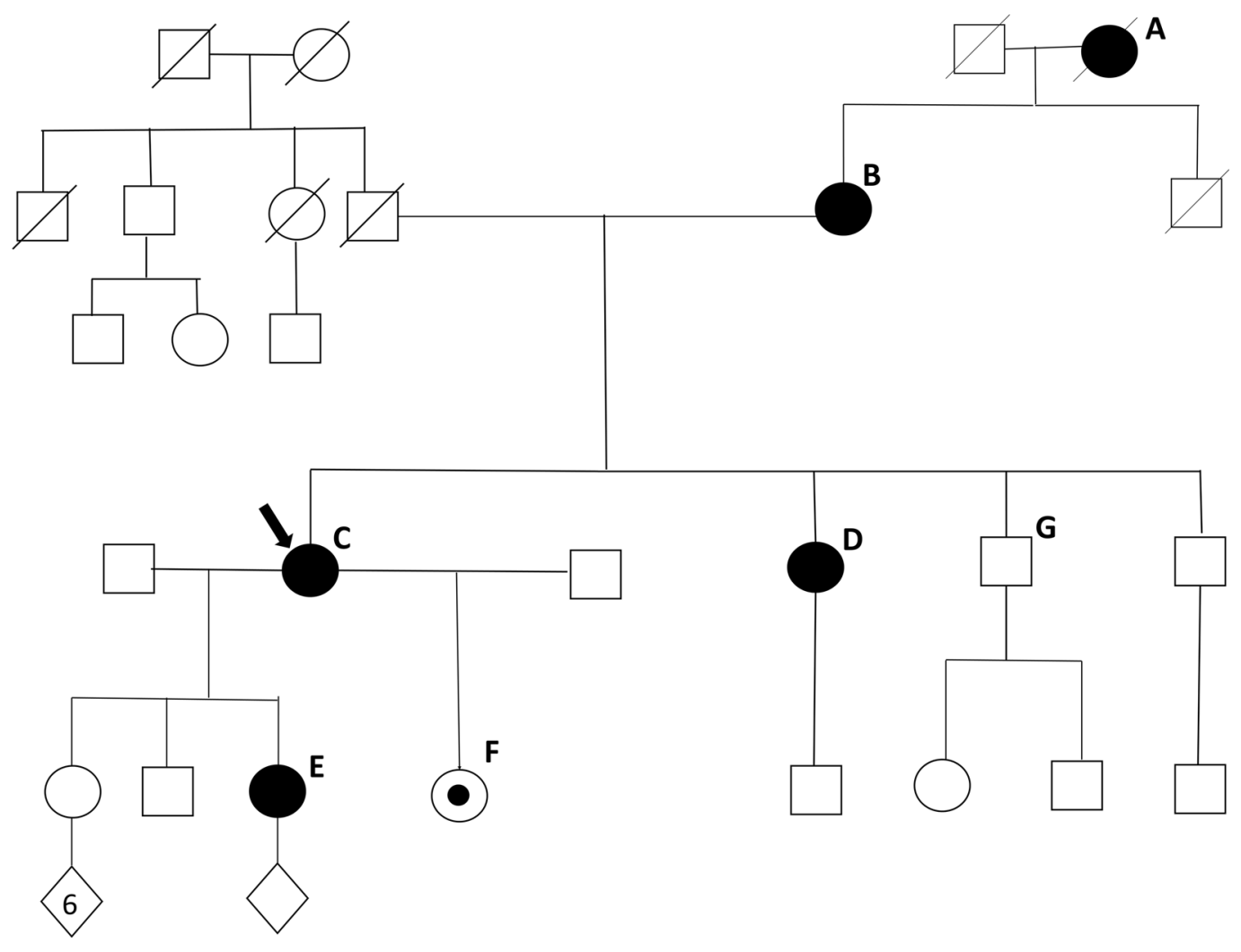

A: breast cancer at $\mathbf{4 3}$ years

B: bilateral breast cancer at $\mathbf{4 2}$ years, history of lung cancer

C: bilateral breast cancer at 55 years; pathogenic variants in BRCA1, BRCA2, CHEK2

D: breast cancer at 35 years

E: breast cancer at 34 years; pathogenic variants in BRCA1 and CHECK2

F: 29 years old, Kartagener's syndrome/situs inversus totalis; pathogenic variants in BRCA1 and CHECK2

G: 60 years old, history of melanoma

Fig. 1 Family pedigree 
primarily in the Ashkenazi Jewish population, among whom $B R C A$ pathogenic variants are ten times more common than in people not of Jewish descent [24, 27-34]. A retrospective study by Rebbeck et al. reported $B R C A 1 / 2$ double heterozygosity in $93(0.3 \%)$ of 32,295 BRCA $1 / 2$ germline pathogenic variant carriers. Of these, 64 patients were diagnosed with breast cancer and 17 patients with epithelial ovarian cancer. Double heterozygotes were more likely to be diagnosed with breast cancer compared to women with only one deleterious $B R C A$ pathogenic variant $(68.1 \%$ vs. $52.0 \%$ for BRCA $1, p=0.002 ; 67.4 \%$ vs. $50.4 \%$ for BRCA2, $p=0.002$ ). Furthermore, this study demonstrated that patients with two inherited pathogenic variants were more likely to develop ovarian cancer compared with those with a BRCA2 pathogenic variant alone ( 16.9 vs. $9.3 \%, p=0.0017)$ [25].

In another retrospective study of a Jewish population of 1191 carriers of BRCA 1 and 2 variants, 22 patients (1.8\%) were double heterozygotes. This investigation suggested a slightly younger age of cancer diagnosis but a similar probability of breast and ovarian cancer development in double heterozygotes compared with single variant carriers [34]. In a study by Leegte et. al, the rate of $B R C A 1 / 2$ double heterozygosity was $0.36 \%$ in $B R C A$ variant carriers; this rate increased to $1.8 \%$ in the sub-population of Askhenazi Jewish patients. In the 34 women with double heterozygosity, there was no significant phenotype differences compared with single variant carriers [27]. Simultaneous BRCA1/2 pathogenic variants were also identified in a systematic review of women with epithelial ovarian cancer. The clinical and prognostic features of individuals with double heterozygosity closely resembled $B R C A I$ variant carriers [29]. In summary, due to the infrequent number of reports, there is wide variability and limited clarity of the clinical and prognostic features associated with having two deleterious germline variants in $B R C A$.

Double heterozygosity in other breast cancer susceptibility genes has also been reported but represents an even rarer occurrence. Relevant to this case study, there have been only a handful of reports of co-existing CHEK2 and $B R C A$ variants. For example, an investigation of germline variants in 5,391 Slavic patients with breast cancer found 17 breast cancer patients in which two pathogenic variants were detected; $4(1.4 \%)$ of these women were found to be $C H E K 2$ and BRCA1 double heterozygotes. The CHEK2 variants included 2 patients with $C H E K 2$ del5395, 1 patient with CHEK2 1100delc and 1 patient with CHEK 2 IVS2 $+1 G>A$. Additionally, one patient in the matched group of 3,884 healthy controls without breast cancer was found to harbor a variant in both $C H E K 2$ and $B R C A 1$ [35]. In another study by Turnbull et al., CHEK2 1100delC was found in $1 / 358$ $(0.28 \%)$ of those with $B R C A 1$ pathogenic variants and $1 / 357$ (0.28\%) BRCA2 pathogenic variant cases [26]. Additionally, co-existing BRCA1 with $C H E K 2$ cases was reported in analysis of a large Polish cohort (7,782 breast cancer and 6,233 control cases), in which patients were assessed for seven founder pathogenic variants in BRCA2 and CHEK2. Double heterozygosity was detected in 15 patients in the breast cancer group and 2 patients in the control group [36]. Conclusions from each of the described case studies of coexisting $C H E K 2$ and $B R C A$ variants suggest no significant increased cancer risk or difference in phenotype beyond what is expected with a single $B R C A$ variant $[26,35,36]$. However, this requires further verification given the limited number of such cases.

Evidenced-based management of individuals with confirmed high penetrance pathogenic variants such as BRCA1 and BRCA 2 can result in earlier detection of breast cancer when early and timely screening using optimal detection methods is applied. Additionally, chemoprophylaxis with endocrine therapy and surgery could result in risk reduction when optimally applied [37-40]. Specifically, risk-reducing bilateral mastectomy has been shown to reduce breast cancer development up to $90 \%$ [39]. Additionally, bilateral salpingo-oophorectomy, typically after completion of childbearing and between the age of 35-40 with BRCAl variants and 40-45 with $B R C A 2$ variants, reduces the risk of ovarian cancer by as much as $80 \%$ and has a mortality benefit [41]; it may also reduce the risk of breast cancer, although these data are inconsistent [41, 42]. For CHEK2, current guidelines recommend screening with annual mammography with consideration of annual breast MRI starting at the age of 40. Additionally, more intensive colon cancer screening is indicated with specific recommendations based on family history of colon cancer [40]. However, there is insufficient evidence to recommend other risk reduction strategies such as bilateral mastectomy, and decision-making should be based on individualized patient and family risk [40].

Decades of research regarding the risk and phenotypic manifestations associated with individual germline variants in hereditary breast cancer has led to the development of informed clinical practice guidelines [2, 40]. However, a challenging aspect of personalized counseling for $B R C A 1 / 2$ as well as $C H E K 2$ pathogenic variants is the relatively large range of cancer risk [14, 36]. This represents an even greater challenge in those with multiple pathogenic variants. In these individuals, the degree of risk is unclear based on the limited reported cases and there are challenges in interpreting management recommendations which apply to single variants. Greater data are needed to understand the clinical implications of an individual harboring multiple variants, such as age of cancer onset, primary tumor characteristics and possibility of multiple primary tumors. A better understanding of the cumulative lifetime cancer risk and whether there is a potential synergistic effect of multiple pathogenic variants on carcinogenesis is additionally necessary. Furthermore, 
it is unclear how other genetic factors such as polygenic risk scores could further modify risk in individuals with co-existing variants, and whether there is resemblance to which these factors impact those with single pathogenic variants [43]. A better understanding of this risk is critical to establish informed clinical decisions and best practices in counseling, screening, risk reduction, and treatment of those with co-existing variants. For patients with double or multiple heterozygosity in known cancer-predisposing genes, our institutional practices consider the highest risk gene variant to guide relevant screening or prevention strategies. For instance, the proband in this case was counseled on breast and ovarian cancer risk based on the presence of a known BRCAl variant. However, she also received counseling for management of risk for other malignancies including colon cancer, pancreatic cancer, and melanoma based on the presence of concurrent pathogenic mutations in BRCA2 and CHEK2.

Based on individualized care and shared decision-making, certain patients with co-existing variants may benefit from more intensive surveillance/screening programs or risk reduction strategies beyond the standard recommendation for single pathogenic variants. It will also be critical to understand whether such interventions may affect survival in this population. Additionally, biologic data on the risks associated with multiple germline variants may influence decisions related to therapeutics, such as use of platinumbased chemotherapy or PARP inhibitors, in those diagnosed with breast and ovarian cancer. Namely, it is unclear whether the efficacy of these DNA damaging agents will be impacted by multiple variants in comparison to those with a single deleterious variant.

This family case report also emphasizes the importance of multi-gene panel testing as the preferred method of genetic analysis in patients at risk for hereditary breast cancer, which is the current standard of care for genetic assessment in the proband. This strategy not only improves efficiency and cost-effectiveness, but may also detect multiple susceptibility genes in an affected individual, as was seen in our case report. Moreover, in individuals known to carry a single pathogenic variant based on phenotype-directed testing, a detailed family and cancer history should be obtained. In these situations, variant analysis should be extended on a case-by-case basis in those with a strong clinical suspicion of manifesting multiple pathogenic variants, such as a strong family history of cancer on both sides. In this family case series, single-site genetic testing for the pathogenic variants found in the proband was pursued in the offspring as there was no known paternal family history of cancer in the offspring. These recommendations are in concordance with the NCCN guidelines [40]. This may impact clinical management as knowledge of multiple susceptibility gene variants in an individual may guide tailored recommendations and modifications in cancer screening and risk reduction approaches.

\section{Conclusion}

Identification of more individuals with multiple co-existing germline variants related to familial breast cancer will facilitate an improved understanding of the phenotypic manifestations of these unique genotypes in family clusters and provide better insight into optimal clinical management practices. There is currently no standard of care for management in patients with co-existing pathogenic variants, and a personalized and tailored approach in genetic counseling, early detection, and risk reduction strategies should be undertaken with the goal to improve cancer and healthrelated outcomes.

Author contributions JS and MK prepared the body of the manuscript. DA, HC, RP, BR, KS, and SS critically reviewed and edited the publication. All the authors endorsed the final form of the manuscript.

Data availability All data are included in the manuscript.

\section{Compliance with ethical standards}

Conflict of interest The authors declare that the research was conducted in the absence of any commercial or financial relationships that could be construed as a potential conflict of interest.

Informed consent Informed consents were obtained from the patients for publication of this report and accompanying images.

Open Access This article is licensed under a Creative Commons Attribution 4.0 International License, which permits use, sharing, adaptation, distribution and reproduction in any medium or format, as long as you give appropriate credit to the original author(s) and the source, provide a link to the Creative Commons licence, and indicate if changes were made. The images or other third party material in this article are included in the article's Creative Commons licence, unless indicated otherwise in a credit line to the material. If material is not included in the article's Creative Commons licence and your intended use is not permitted by statutory regulation or exceeds the permitted use, you will need to obtain permission directly from the copyright holder. To view a copy of this licence, visit http://creativecommons.org/licenses/by/4.0/.

\section{References}

1. Tung N, Lin NU, Kidd J, Allen BA, Singh N, Wenstrup RJ et al (2016) Frequency of germline mutations in 25 cancer susceptibility genes in a sequential series of patients with breast cancer. $\mathrm{J}$ Clin Oncol 34(13): 1460-1468

2. Tung NM, Boughey JC, Pierce LJ, Robson ME, Bedrosian I, Dietz JR et al (2020) Management of Hereditary Breast Cancer: American Society of Clinical Oncology, American Society for Radiation 
Oncology, and Society of Surgical Oncology Guideline. J Clin Oncol 38(18):2080-2106

3. Locatelli I, Lichtenstein P, Yashin AI (2004) The heritability of breast cancer: a Bayesian correlated frailty model applied to Swedish twins data. Twin Res 7(2):182-191

4. Moller S, Mucci LA, Harris JR, Scheike T, Holst K, Halekoh U et al (2016) The heritability of breast cancer among women in the Nordic Twin Study of Cancer. Cancer Epidemiol Biomark Prev 25(1):145-150

5. Couch FJ, Shimelis H, Hu C, Hart SN, Polley EC, Na J et al (2017) Associations between cancer predisposition testing panel genes and breast cancer. JAMA Oncol 3(9):1190-1196

6. Buys SS, Sandbach JF, Gammon A, Patel G, Kidd J, Brown KL et al (2017) A study of over 35,000 women with breast cancer tested with a 25-gene panel of hereditary cancer genes. Cancer 123(10):1721-1730

7. Shattuck-Eidens D, Oliphant A, McClure M, McBride C, Gupte J, Rubano T et al (1997) BRCA1 sequence analysis in women at high risk for susceptibility mutations: risk factor analysis and implications for genetic testing. JAMA 278(15):1242-1250

8. Ford D, Easton DF, Stratton M, Narod S, Goldgar D, Devilee P et al (1998) Genetic heterogeneity and penetrance analysis of the BRCA1 and BRCA2 genes in breast cancer families. The Breast Cancer Linkage Consortium. Am J Hum Genet 62(3):676-689

9. Gonzalez-Angulo AM, Timms KM, Liu S, Chen H, Litton JK, Potter J et al (2011) Incidence and outcome of BRCA mutations in unselected patients with triple receptor-negative breast cancer. Clin Cancer Res 17(5):1082-1089

10. McClain MR, Palomaki GE, Nathanson KL, Haddow JE (2005) Adjusting the estimated proportion of breast cancer cases associated with BRCA1 and BRCA2 mutations: public health implications. Genet Med 7(1):28-33

11. Roa BB, Boyd AA, Volcik K, Richards CS (1996) Ashkenazi Jewish population frequencies for common mutations in BRCA1 and BRCA2. Nat Genet 14(2):185-187

12. Hartge P, Struewing JP, Wacholder S, Brody LC, Tucker MA (1999) The prevalence of common BRCA1 and BRCA2 mutations among Ashkenazi Jews. Am J Hum Genet 64(4):963-970

13. Gudmundsdottir K, Ashworth A (2006) The roles of BRCA1 and BRCA2 and associated proteins in the maintenance of genomic stability. Oncogene 25(43):5864-5874

14. Kuchenbaecker KB, Hopper JL, Barnes DR, Phillips KA, Mooij TM, Roos-Blom MJ et al (2017) Risks of breast, ovarian, and contralateral breast cancer for BRCA1 and BRCA2 mutation carriers. JAMA 317(23):2402-2416

15. Chen S, Parmigiani G (2007) Meta-analysis of BRCA1 and BRCA2 penetrance. J Clin Oncol 25(11):1329-1333

16. Consortium C-BC (2002) Low-penetrance susceptibility to breast cancer due to CHEK2* 1100delC in noncarriers of BRCA1 or BRCA2 mutations. Nat Genet 31(1):55

17. Consortium CBCC-C (2004) CHEK2* 1100delC and susceptibility to breast cancer: a collaborative analysis involving 10,860 breast cancer cases and 9,065 controls from 10 studies. Am J Hum Genet 74(6): 1175-1182

18. Easton DF, Pharoah PD, Antoniou AC, Tischkowitz M, Tavtigian SV, Nathanson KL et al (2015) Gene-panel sequencing and the prediction of breast-cancer risk. N Engl J Med 372(23):2243-2257

19. Schmidt MK, Tollenaar RA, de Kemp SR, Broeks A, Cornelisse CJ, Smit VT et al (2007) Breast cancer survival and tumor characteristics in premenopausal women carrying the CHEK2*1100delC germline mutation. J Clin Oncol 25(1):64-69

20. Cybulski C, Wokołorczyk D, Jakubowska A, Huzarski T, Byrski $\mathrm{T}$, Gronwald J et al (2011) Risk of breast cancer in women with a CHEK 2 mutation with and without a family history of breast cancer. J Clin Oncol 29(28):3747-3752
21. Bernstein JL, Teraoka SN, John EM, Andrulis IL, Knight JA, Lapinski $\mathrm{R}$ et al (2006) The CHEK2* 1100delC allelic variant and risk of breast cancer: screening results from the Breast Cancer Family Registry. Cancer Epidemiol Prev Biomark 15(2):348-352

22. Stolz A, Ertych N, Bastians H (2011) Tumor suppressor CHK2: regulator of DNA damage response and mediator of chromosomal stability. Clin Cancer Res 17(3):401-405

23. Xiang HP, Geng XP, Ge WW, Li H (2011) Meta-analysis of CHEK2 1100delC variant and colorectal cancer susceptibility. Eur J Cancer 47(17):2546-2551

24. Landrum MJ, Lee JM, Benson M, Brown GR, Chao C, Chitipiralla $S$ et al (2018) ClinVar: improving access to variant interpretations and supporting evidence. Nucleic Acids Res 46(D1):D1062-D1067

25. Rebbeck TR, Friebel TM, Mitra N, Wan F, Chen S, Andrulis IL et al (2016) Inheritance of deleterious mutations at both BRCA1 and BRCA2 in an international sample of 32,295 women. Breast Cancer Res 18(1):112

26. Turnbull C, Seal S, Renwick A, Warren-Perry M, Hughes D, Elliott A et al (2012) Gene-gene interactions in breast cancer susceptibility. Hum Mol Genet 21(4):958-962

27. Leegte B, van der Hout AH, Deffenbaugh AM, Bakker MK, Mulder IM, ten Berge A et al (2005) Phenotypic expression of double heterozygosity for BRCA1 and BRCA2 germline mutations. J Med Genet 42(3):e20

28. Cline MS, Liao RG, Parsons MT, Paten B, Alquaddoomi F, Antoniou A et al (2018) BRCA challenge: BRCA exchange as a global resource for variants in BRCA1 and BRCA2. PLoS Genet 14(12):e1007752

29. Le Page C, Rahimi K, Rodrigues M, Heinzelmann-Schwarz V, Recio N, Tommasi S et al (2020) Clinicopathological features of women with epithelial ovarian cancer and double heterozygosity for BRCA1 and BRCA2: a systematic review and case report analysis. Gynecol Oncol 156(2):377-386

30. Vietri MT, Molinari AM, Caliendo G, De Paola ML, Giovanna D, Gambardella AL et al (2013) Double heterozygosity in the BRCA1 and BRCA2 genes in Italian family. Clin Chem Lab Med 51(12):2319-2324

31. Liao Y, Tu C, Song X, Cai L (2020) Case report: analysis of BRCA1 and BRCA2 gene mutations in a hereditary ovarian cancer family. J Assist Reprod Genet 37(6):1489-1495

32. Cardoso FC, Goncalves S, Mele PG, Liria NC, Sganga L, Diaz Perez I et al (2018) BRCA1 and BRCA2 mutations and clinical interpretation in 398 ovarian cancer patients: comparison with breast cancer variants in a similar population. Hum Genomics 12(1):39

33. Heidemann S, Fischer C, Engel C, Fischer B, Harder L, Schlegelberger B et al (2012) Double heterozygosity for mutations in BRCA1 and BRCA2 in German breast cancer patients: implications on test strategies and clinical management. Breast Cancer Res Treat 134(3):1229-1239

34. Lavie O, Narod S, Lejbkowicz F, Dishon S, Goldberg Y, Gemer $\mathrm{O}$ et al (2011) Double heterozygosity in the BRCA1 and BRCA2 genes in the Jewish population. Ann Oncol 22(4):964-966

35. Sokolenko AP, Bogdanova N, Kluzniak W, Preobrazhenskaya EV, Kuligina ES, Iyevleva AG et al (2014) Double heterozygotes among breast cancer patients analyzed for BRCA1, CHEK2, ATM, NBN/NBS1, and BLM germ-line mutations. Breast Cancer Res Treat 145(2):553-562

36. Cybulski C, Górski B, Huzarski T, Byrski T, Gronwald J, Debniak $T$ et al (2009) Effect of CHEK2 missense variant I157T on the risk of breast cancer in carriers of other CHEK 2 or BRCA 1 mutations. J Med Genet 46(2):132-135

37. Burke W, Daly M, Garber J, Botkin J, Kahn MJE, Lynch P et al (1997) Recommendations for follow-up care of individuals with 
an inherited predisposition to cancer: II. BRCA1 and BRCA2. JAMA 277(12):997-1003

38. Warner E, Causer PA (2005) MRI surveillance for hereditary breast-cancer risk. The Lancet 365(9473):1747-1749

39. Hartmann LC, Sellers TA, Schaid DJ, Frank TS, Soderberg CL, Sitta DL et al (2001) Efficacy of bilateral prophylactic mastectomy in BRCA1 and BRCA2 gene mutation carriers. J Natl Cancer Inst 93(21):1633-1637

40. Daly MB, Pilarski R, Yurgelun MB, Berry MP, Buys SS, Dickson $P$ et al (2020) NCCN guidelines insights: genetic/familial highrisk assessment: breast, ovarian, and pancreatic, version 1.2020. J Natl Compr Canc Netw. 18(4):380-391

41. Rebbeck TR, Kauff ND, Domchek SM (2009) Meta-analysis of risk reduction estimates associated with risk-reducing salpingooophorectomy in BRCA1 or BRCA2 mutation carriers. J Natl Cancer Inst 101(2):80-87
42. Kotsopoulos J, Huzarski T, Gronwald J, Singer CF, Moller P, Lynch HT et al (2017) Bilateral oophorectomy and breast cancer risk in BRCA1 and BRCA2 mutation carriers. J Natl Cancer Inst 109(1)

43. Gallagher S, Hughes E, Wagner S, Tshiaba P, Rosenthal E, Roa BB et al (2020) Association of a polygenic risk score with breast cancer among women carriers of high- and moderate-risk breast cancer genes. JAMA Netw Open 3(7):e208501

Publisher's Note Springer Nature remains neutral with regard to jurisdictional claims in published maps and institutional affiliations. 